ANNALES

POLONICI MATHEMATICI

LXVI (1997)

\title{
Covariant differential operators and Green's functions
}

\author{
by Miroslav Engliš (Praha) and JaAk Peetre (Lund)
}

This paper is dedicated to the memory of Wtodzimierz Mlak, who was one of my earliest mathematical acquaintances. We met in 1961/62 when we both were research associates at the University of Maryland. I remember vividly Wtodzimierz taking driving lessons from his mentor R. E. Fullerton, who died in a car accident in Spain some years later. Much later we spoke once over the phone but did not see each other again. The present paper does perhaps not reflect much of Mlak's mathematical interests, but at least it comes in some sense close to the type of PDE that was cultivated at College Park at the time-I am now referring to the late Alexander Weinstein and his school.

$$
\text { J. P. }
$$

Abstract. The basic idea of this paper is to use the covariance of a partial differential operator under a suitable group action to determine suitable associated Green's functions. For instance, we offer a new proof of a formula for Green's function of the $m$ th power $\Delta^{m}$ of the ordinary Laplace's operator $\Delta$ in the unit disk $\mathbb{D}$ found in a recent paper (HaymanKorenblum, J. Anal. Math. 60 (1993), 113-133). We also study Green's functions associated with $m$ th powers of the Poincaré invariant Laplace operator $\boldsymbol{\Delta}$. It turns out that they can be expressed in terms of certain special functions of which the dilogarithm $(m=2)$ and the trilogarithm $(m=3)$ are the simplest instances. Finally, we establish a relationship between $\Delta^{m}$ and $\Delta$ : the former is up to conjugation a polynomial of the latter.

0. Introduction. In this paper we investigate Green's functions associated with some covariant partial differential operators. The underlying idea is simple. Let a manifold be given on which there acts a transitive group of

1991 Mathematics Subject Classification: 35C10, 35J40.

Key words and phrases: covariant differential operator, Laplace operator, Green's function, Hayman-Korenblum fomula, Bojarski's theorem, Bol's lemma, covariant CauchyRiemann operator, dilogarithm, trilogarithm, general nonsense.

The first author sponsored by the "Civilingenjör Gustaf Sigurd Magnusons fond för främjande av vetenskapen inom ämnet matematik" of the Royal Swedish Academy of Sciences (Kungl. Vetenskapsakademien) and also in part by GA AV ČR grant No. 119106. 
transformations and consider on it a partial differential operator covariant with respect to this group action. In order to determine a Green's function for this differential operator it suffices, in view of the covariance and the transitivity, to find it at some fixed point, usually the "origin". (By Green's function we mean, informally, a function $G=G(z, w)$ which, fixing the second argument $w$ and denoting the differential operator by $\mathfrak{L}$ and the delta function at $w$ by $\delta$, satisfies the differential equation $\mathfrak{L} G=\delta$ and, in addition, appropriate boundary conditions.)

For instance, in Section 1 we give, as a warm-up, a new proof of a formula for Green's function for the $m$ th power $\Delta^{m}$ of the ordinary Laplace's operator $\Delta$ in the unit disk $\mathbb{D}$ to be found in a recent paper by HaymanKorenblum [7]. The formula itself is valid for any number of (real) variables but to fix ideas we restrict ourselves, as also in most of the rest of this paper, to the two-dimensional case. The covariance of the operator in this case is due to Bojarski [4]. Another point made in this connection (Section 2) is a perhaps novel computation of the constant involved. Again the approach used is applicable in other cases as well, and in a remark we indicate a proof of the Bochner-Martinelli formula in several complex variables.

In Sections 3 and 4 we put into play the Möbius invariant Laplace operator $\boldsymbol{\Delta}$ on $\mathbb{D}$ equipped with the usual hyperbolic metric (that is, the LaplaceBeltrami operator with respect to this metric). In Section 3 we determine the corresponding Green's function for the operator $\Delta^{2}$, whereas Section 4 is concerned with the generalization of this result to higher powers, the operators $\boldsymbol{\Delta}^{m}, m>2$; however, in the latter case our result is reasonably complete only in the simplest case $m=3$. It turns out - and this is something quite unexpected - that the result comes in terms of certain special functions, so-called logarithmic functions, the dilogarithm $\mathrm{Li}_{2}$ if $m=2$ and the trilogarithm $\mathrm{Li}_{3}$ if $m=3$. It is reasonable to expect that the answer also in the general case $(m>3)$, not yet settled, will involve more general logarithmic functions related to the polylogarithm $\mathrm{Li}_{m}$.

In Section 5 we establish a connection between the iterated operators $\Delta^{m}$ and the invariant Laplacean $\boldsymbol{\Delta}$, by showing that the former are polynomials of the latter.

Finally, in Section 6 we discuss briefly, without going into too much detail, to what extent the results of this paper can be extended to the case of several complex variables.

1. The Hayman-Korenblum formula. We seek Green's function for the operator $\Delta^{m}$ in the unit disc $\mathbb{D}$ with Dirichlet boundary conditions (vanishing of the normal derivatives up to order $m-1$ ).

It is easy to see that $\Delta^{m}$ has the fundamental solution

$$
F(z)=c r^{2 m-2} \log r^{2} \quad(r=|z|) .
$$


Here $c=c_{m}$ is a constant whose exact value will be determined in Section 2 . This suggests that the Green's function taken at the origin be of the form

$$
G(z)=G(z, 0)=c\left(r^{2 m-2} \log r^{2}+\sum_{i=0}^{m-1} \alpha_{i}\left(1-r^{2}\right)^{i}\right) .
$$

Introduce the variable $t=r^{2}$. We must determine the coefficients $\alpha_{i}$ in such a way that the function

$$
g=t^{m-1} \log t+\sum_{i=0}^{m-1} \alpha_{i}(1-t)^{i}
$$

vanishes at $t=1$ together with its derivatives up to order $m-1$.

Lemma 1. Fix a number $\nu$. Put $f=t^{\nu} \log t$ and write $D=\frac{d}{d t}$. Then

$$
D^{k} f=\nu(\nu-1) \ldots(\nu-k+1) t^{\nu-k} \log t+a_{k} t^{\nu-k} \quad(k=0,1,2, \ldots),
$$

where

$$
a_{k}=\sum_{s=0}^{k-1} \underbrace{\nu(\nu-1) \ldots(\widehat{\nu-s}) \ldots(\nu-k+1)}_{k-1 \text { factors }}
$$

and the sign ${ }^{-}$means that the corresponding factor is omitted in the product. If $\nu \neq 1, \ldots, k-1$ then (2) can alternatively be written as

$$
a_{k}=\nu(\nu-1) \ldots(\nu-k+1) \sum_{s=0}^{k-1} \frac{1}{\nu-s} .
$$

Pro of (by induction over $k$ ). Formula (1) is trivial for $k=0$ (with $\left.a_{0}=0\right)$. Suppose (1) holds true for the index $k$ with some constant $a_{k}$. Then we find

$$
\begin{aligned}
D^{k+1} f= & \nu(\nu-1) \ldots(\nu-k+1)(\nu-k) t^{\nu-k-1} \log t \\
& +\nu(\nu-1) \ldots(\nu-k+1) t^{\nu-k-1}+a_{k}(\nu-k) t^{\nu-k-1} .
\end{aligned}
$$

This gives (1) for the index $k+1$ if we put

$$
a_{k+1}=(\nu-k) a_{k}+\nu(\nu-1) \ldots(\nu-k+1) .
$$

The recursion (4) is likewise solved by induction. Suppose thus that $a_{k}$ satisfies (2). Then (4) gives

$$
\begin{aligned}
a_{k+1}= & \sum_{s=0}^{k-1} \nu(\nu-1) \ldots(\widehat{\nu-s}) \ldots(\nu-k+1)(\nu-k) \\
& +\nu(\nu-1) \ldots(\nu-k+1) \\
= & \sum_{s=0}^{k} \nu(\nu-1) \ldots(\widehat{\nu-s}) \ldots . .(\nu-k),
\end{aligned}
$$


which is (2) with $k$ replaced by $k+1$. The truth of (3) is obvious. find

Return to the function $g$. Using Lemma 1 with $\nu=m-1$, from (1) we

$$
\begin{aligned}
D^{k} g= & (m-1)(m-2) \ldots(m-1-k+1) t^{m-k-1} \log t+a_{k} t^{m-1-k} \\
& +\sum_{i=0}^{m-1} \alpha_{i} i(i-1) \ldots(i-k+1)(-1)^{k}(1-t)^{i-k}
\end{aligned}
$$

$(k=0,1, \ldots, m-1)$, where now (in view of (3) with $\nu=m-1)$

$$
a_{k}=\underbrace{(m-1)(m-2) \ldots(m-1-k+1)}_{k-1 \text { factors }} \sum_{s=0}^{k-1} \frac{1}{m-1-s} .
$$

Putting $t=1$ gives

$$
a_{k}+\alpha_{k} k !(-1)^{k}=0
$$

or, solving out and using (5),

$$
\alpha_{k}=\frac{(-1)^{k+1}}{k !} a_{k}=(-1)^{k+1}\left(\begin{array}{c}
m-1 \\
k
\end{array}\right) \sum_{s=m-k}^{m-1} \frac{1}{s} .
$$

We have thus proved that

$$
G(z)=G(z, 0)=c\left(r^{2 m-2} \log r^{2}+\sum_{i=0}^{m-1} \alpha_{i}\left(1-r^{2}\right)^{i}\right)
$$

with the $\alpha_{i}$ 's given by $(6)$.

Recall next that if $u$ is any solution of the equation $\Delta^{m} u=0$ and $\omega(z)=(a z+b) /(c z+d)$ is a Möbius transform determined by the unimodular matrix $\left(\begin{array}{l}a b \\ c d\end{array}\right)$ (unimodular means that $a d-b c=1$ ) then the function

$$
v(z)=|e(z)|^{2(m-1)} u(\omega(z))
$$

is likewise a solution of the same equation, where we have put $e(z)=c z+d$.

R e mark 1. The simplest proof of this fact, which is a special case of a theorem of Bojarski [4] valid in any number of dimensions, goes via "Bol's lemma" (cf. [6], where several proofs of this elementary result are given) to the effect that if $v(z)$ is any function and if we put

$$
v_{1}(z)=(e(z))^{m-1} v(\omega(z))
$$

then

and a similar formula holds with the function

$$
\frac{\partial^{m} v_{1}(z)}{\partial z^{m}}=(e(z))^{-m-1} \frac{\partial^{m} v(\omega(z))}{\partial z^{m}},
$$

$$
v_{2}(z)=(\overline{e(z)})^{m-1} v(\omega(z))
$$


and the operator $\frac{\partial}{\partial \bar{z}}$ (instead of $\left.\frac{\partial}{\partial d} z\right)$. We also use the fact that

$$
\Delta=4 \frac{\partial^{2}}{\partial z \partial \bar{z}}
$$

which will do us great service also in what follows.

Now let $w$ be any point in the disc $\mathbb{D}$ and choose $\omega$ such that $\omega(w)=0$. Then it follows that

$$
G(z, w)=G(\omega(z), 0)|e(z)|^{2(m-1)}|e(w)|^{2(m-1)} .
$$

In particular, we can take $\omega(z)=(z-w) /(1-z \bar{w})$. The corresponding matrix is not $\left(\begin{array}{cc}1 & -w \\ -\bar{w} & 1\end{array}\right)$, because the latter is not a unimodular matrix. But this matrix divided by $\left(1-|w|^{2}\right)^{1 / 2}$ is unimodular; then

$$
c=\frac{-\bar{w}}{\left(1-|w|^{2}\right)^{1 / 2}}, \quad d=\frac{1}{\left(1-|w|^{2}\right)^{1 / 2}},
$$

implying that

$$
\begin{aligned}
& e(z)=c z+d=\frac{1-z \bar{w}}{\left(1-|w|^{2}\right)^{1 / 2}}, \\
& e(w)=c w+d=\left(1-|w|^{2}\right)^{1 / 2} .
\end{aligned}
$$

By (7) this gives

$$
\begin{aligned}
G(z, w)= & c\left(|z-w|^{2 m-2} \log \left|\frac{z-w}{1-z \bar{w}}\right|^{2}\right. \\
& \left.+\sum_{i=0}^{m-1} \alpha_{i}\left(1-|z|^{2}\right)^{i}\left(1-|w|^{2}\right)^{i}|1-z \bar{w}|^{2(m-1-i)}\right)
\end{aligned}
$$

where we have also taken account of the well known identity

$$
|1-\bar{z} w|^{2}-|z-w|^{2}=\left(1-|z|^{2}\right)\left(1-|w|^{2}\right) .
$$

The coefficients $\alpha_{i}$ in (9) are still those given by (6).

R e mark 2. It is easy to see that (9) agrees, in the case of dimension 2, with the formula given in [7] (formula (1.6), p. 115). In the two-dimensional case the latter formula takes the form

$$
\begin{aligned}
G(z, w)= & c \cdot(-1)^{m}(m-1) ! \\
& \times \sum_{\nu=0}^{\infty} \frac{1}{(\nu+1)(\nu+2) \ldots(\nu+m)} \frac{\left(1-|z|^{2}\right)^{m+\nu}\left(1-|w|^{2}\right)^{m+\nu}}{|1-z \bar{w}|^{2+2 \nu}}
\end{aligned}
$$


where the right hand side can also be written in a perhaps more conspicuous form as

$$
\begin{aligned}
& c \cdot(-1)^{m}(m-1) !|1-z \bar{w}|^{2(m-1)} \\
& \quad \times \sum_{\nu=0}^{\infty} \frac{1}{(\nu+1)(\nu+2) \ldots(\nu+m)}\left(\frac{\left(1-|z|^{2}\right)\left(1-|w|^{2}\right)}{|1-z \bar{w}|^{2}}\right)^{\nu+m} .
\end{aligned}
$$

In view of the covariance it suffices to take $w=0$, as always. Then it is a question about the identity

$$
\begin{aligned}
g & =t^{m-1} \log t+\sum_{i=1}^{m-1} \alpha_{i}(1-t)^{i} \\
& =(-1)^{m}(m-1) ! \sum_{\nu=0}^{\infty} \frac{(1-t)^{\nu+m}}{(\nu+1)(\nu+2) \ldots(\nu+m)},
\end{aligned}
$$

where the $\alpha_{i}$ 's are given by (6).

Pro of of (12). Indeed, by the construction of the $\alpha_{i}$ 's, the Taylor coefficients of $g$ at $t=1$ vanish up to order $m-1$. On the other hand, Lemma 1 also gives

$$
\begin{gathered}
D^{m} g=(m-1) ! t^{-1}, \\
D^{m+1} g=-(m-1) ! t^{-2}, \\
D^{m+2} g=(m-1) ! 2 t^{-3} \quad \text { etc. }
\end{gathered}
$$

and quite generally

$$
D^{m+\mu} g=(-1)^{\mu}(m-1) ! \mu ! t^{-1-\mu} .
$$

Therefore, putting $t=1$, we also obtain the remaining Taylor coefficients and so the identity follows.

It is, apparently, a matter of taste which of the two formulae, (9) or (11), is the simplest. The authors of [7] say that "it seems surprising that a simple formula yields [the Green's function] for all $m$ ". One virtue of (11) is that it directly implies the constancy of sign. Hayman-Korenblum further offer the conjecture, even in the higher-dimensional case, that "balls are the only domains ... whose Green's functions ... have constant sign ... for all positive integers $m$ ". Actually, the problem of finding Green's function for the operator $\Delta^{m}$ was in principle already settled by Tommaso Boggio [3] in 1905. This author also pointed out the phenomenon of constancy of sign. According to Hedenmalm [8], Boggio in another, earlier paper [2] had 
raised the question of whether Green's function of the operator $\Delta^{m}$ for an arbitrary planar domain is of constant sign, a question often referred to as Hadamard's conjecture.

2. Evaluation of the constant. We must now determine the constant $c=c_{m}$ of Section 1 such that $\Delta^{m} F=\delta$, which will then imply that $\Delta_{z}^{m} G=\delta_{w}$ (delta function at the origin and at the point $w$ respectively).

Let us begin by describing our procedure in the case $m=1$. The guiding idea is to "smoothen" the function

$$
F(z)=c \log |z|^{2}
$$

by replacing it with the function

$$
F_{\varepsilon}(z)=c \log \left(|z|^{2}+\varepsilon^{2}\right),
$$

where $\varepsilon>0$ is a parameter which is eventually going to be 0 . Differentiating yields

$$
\frac{\partial F_{\varepsilon}}{\partial \bar{z}}=c \frac{z}{|z|^{2}+\varepsilon^{2}}
$$

Continuing the differentiation and remembering formula (8) of Section 1 then gives

$$
\Delta F_{\varepsilon}=c \cdot 4 \frac{\partial}{\partial z}\left(\frac{z}{|z|^{2}+\varepsilon^{2}}\right)=c \cdot 4\left(\frac{1}{|z|^{2}+\varepsilon^{2}}-\frac{z \bar{z}}{\left(|z|^{2}+\varepsilon^{2}\right)^{2}}\right)=c \cdot 4 \frac{\varepsilon^{2}}{\left(|z|^{2}+\varepsilon^{2}\right)^{2}} .
$$

It follows that if we let $\varepsilon \rightarrow 0$ then the right hand side converges in the sense of distributions to a multiple of $\delta$, namely the value of the integral on the right hand side extended over $\mathbb{C}$. We have to choose $c$ so that this value becomes unity. As the integral clearly is independent of $\varepsilon$, it suffices to take $\varepsilon=1$. We then find

$$
\int_{\mathbb{C}} \frac{d x d y}{\left(|z|^{2}+1\right)^{2}}=2 \pi \int_{0}^{\infty} \frac{r d r}{\left(r^{2}+1\right)^{2}}=\pi \int_{0}^{\infty} \frac{d t}{(t+1)^{2}}=\pi\left[-\frac{1}{1+t}\right]_{0}^{\infty}=\pi
$$

Hence it follows that $c=c_{1}=1 /(4 \pi)$.

Remark 1. Usually one writes $\log r$ in place of $\log r^{2}$, as here, and then the constant is $1 /(2 \pi)$.

Consider now the general case. If

$$
F(z)=c|z|^{2(m-1)} \log |z|^{2}
$$

we consider the "smooth" version

$$
F_{\varepsilon}(z)=c\left(|z|^{2}+\varepsilon^{2}\right)^{m-1} \log \left(|z|^{2}+\varepsilon^{2}\right) .
$$


Using Lemma 1 of Section 1 (we consider for a moment $\bar{z}$ as an independent variable!) gives

$$
\frac{\partial^{m} F_{\varepsilon}}{\partial \bar{z}^{m}}=c \cdot(m-1) ! \frac{z^{m}}{|z|^{2}+\varepsilon^{2}} .
$$

Continuing the differentiation and using Leibniz's formula (product rule) we find that

$$
\begin{aligned}
\Delta^{m} F_{\varepsilon}= & c \cdot 4^{m}(m-1) ! \frac{\partial^{m}}{\partial z^{m}}\left(\frac{z^{m}}{|z|^{2}+\varepsilon^{2}}\right) \\
= & c \cdot 4^{m}(m-1) ! \sum_{k=0}^{m}(-1)^{k}\left(\begin{array}{c}
m \\
k
\end{array}\right) \frac{k ! \bar{z}^{k}}{\left(|z|^{2}+\varepsilon^{2}\right)^{1+k}} \\
& \times m(m-1) \ldots(m-(m-k)+1) z^{m-(m-k)} \\
= & c \cdot 4^{m} m !(m-1) ! \sum_{k=0}^{m}(-1)^{k}\left(\begin{array}{c}
m \\
k
\end{array}\right) \frac{|z|^{2 k}}{\left(|z|^{2}+\varepsilon^{2}\right)^{1+k}} \\
= & c \cdot 4^{m} m !(m-1) ! \frac{1}{|z|^{2}+\varepsilon^{2}}\left(1-\frac{|z|^{2}}{|z|^{2}+\varepsilon^{2}}\right)^{m} \\
= & c \cdot 4^{m} m !(m-1) ! \frac{\varepsilon^{2 m}}{\left(|z|^{2}+\varepsilon^{2}\right)^{m+1}} .
\end{aligned}
$$

As in the case $m=1$ we conclude that $\Delta^{m} F_{\varepsilon}$ tends to a multiple of $\delta$. In order that this multiple be unity, the integral of this function must again be unity. We have

$$
\begin{aligned}
\int_{\mathbb{C}} \frac{d x d y}{\left(|z|^{2}+1\right)^{1+m}} & =2 \pi \int_{0}^{\infty} \frac{r d r}{\left(r^{2}+1\right)^{1+m}} \\
& =\pi \int_{0}^{\infty} \frac{d t}{\left(t^{2}+1\right)^{1+m}}=\pi\left[\frac{-1}{m} \frac{1}{(1+t)^{m}}\right]_{0}^{\infty}=\frac{\pi}{m} .
\end{aligned}
$$

Thus we conclude that

$$
c=c_{m}=\frac{1}{4^{m}(m-1) !^{2} \pi} .
$$

In particular, we have

$$
\begin{gathered}
c_{1}=\frac{1}{4 \pi}, \quad c_{2}=\frac{1}{16 \pi}, \quad c_{3}=\frac{1}{64 \cdot 4 \pi}, \\
c_{4}=\frac{1}{256 \cdot 36 \pi}, \quad c_{5}=\frac{1}{1024 \cdot 576 \pi} \quad \text { etc. }
\end{gathered}
$$

We may summarize what is proven up to now as follows.

Scholium 1. In order to indicate the dependence on $m$, let us in our notation for Green's function append a subscript, thus writing $G=G_{m}$. 
Then we have

$$
\begin{aligned}
G_{1}= & \frac{1}{4 \pi} \log \left|\frac{z-w}{1-z \bar{w}}\right|^{2}, \\
G_{2}= & \frac{1}{16 \pi}\left(|z-w|^{2} \log \left|\frac{z-w}{1-z \bar{w}}\right|^{2}+\left(1-|z|^{2}\right)\left(1-|w|^{2}\right)\right), \\
G_{3}= & \frac{1}{256 \pi}\left(|z-w|^{4} \log \left|\frac{z-w}{1-z \bar{w}}\right|^{2}\right. \\
& \left.+\left(1-|z|^{2}\right)\left(1-|w|^{2}\right)|1-z \bar{w}|^{2}-\frac{3}{2}\left(1-|z|^{2}\right)^{2}\left(1-|w|^{2}\right)^{2}\right), \\
G_{4}= & \frac{1}{9216 \pi}\left(|z-w|^{6} \log \left|\frac{z-w}{1-z \bar{w}}\right|^{2}+\left(1-|z|^{2}\right)\left(1-|w|^{2}\right)|1-z \bar{w}|^{4}\right. \\
& \left.-\frac{5}{2}\left(1-|z|^{2}\right)^{2}\left(1-|w|^{2}\right)^{2}|1-z \bar{w}|^{2}+\frac{11}{6}\left(1-|z|^{2}\right)^{3}\left(1-|w|^{2}\right)^{3}\right),
\end{aligned}
$$

and so forth.

Remark 2 (on formulae of the Bochner-Martinelli type). The method used here has general character and may also be applied in higher-dimensional cases. It is particularly useful in the context of several complex variables. As an illustration let us indicate a proof of the classical Bochner-Martinelli formula itself. To this end consider in $\mathbb{C}^{n}$ the following differential form of bidegree $(n, n-1)$ :

$$
k=\sum_{i=1}^{n}(-1)^{i-1} k^{i} d \bar{z}^{1} \wedge \ldots \wedge \widehat{d \bar{z}^{i}} \wedge \ldots \wedge d \bar{z}^{n} \wedge d z^{1} \wedge \ldots \wedge d z^{n},
$$

where

$$
k^{i}=c \cdot \frac{\bar{z}^{i}}{|z|^{2}}
$$

and $c=c_{n}$ is a suitable constant. (Points in $\mathbb{C}^{n}$ are denoted by $z=$ $\left(z^{1}, \ldots, z^{n}\right),|z|^{2}=\left|z^{1}\right|^{2}+\ldots+\left|z^{n}\right|^{2}$.) Indeed, as we shall now indicate, it is possible to choose $c$ in such a manner that

$$
d k=\delta d \bar{z}^{1} \wedge \ldots \wedge \ldots \wedge d \bar{z}^{n} \wedge d z^{1} \wedge \ldots \wedge d z^{n},
$$

or equivalently, if we want to avoid differential forms and exterior derivatives, in terms of a divergence:

$$
\sum_{i=1}^{n} \frac{\partial k^{i}}{\partial \bar{z}^{i}}=\delta
$$


In order to prove (1) and (2) one just has to introduce the regularization

$$
k_{\varepsilon}^{i}=c \cdot \frac{\bar{z}^{i}}{|z|^{2}+\varepsilon^{2}} \quad(\varepsilon>0)
$$

and proceed as before to compute the divergence, afterwards making $\varepsilon=0$. In particular, we can determine the constant:

$$
c=c_{n}=\frac{n !}{\pi^{n}} .
$$

But (1) (or (2)) is just the infinitesimal form of the Bochner-Martinelli formula. Integrating we find the corresponding finite formula:

$$
\begin{aligned}
& f(z)=\frac{(-1)^{n(n-1) / 2} n !}{(2 \pi i)^{n}}
\end{aligned}
$$

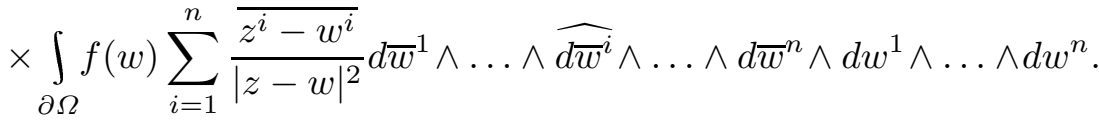

Here $\Omega$ is any bounded domain in $\mathbb{C}^{n}$ with smooth boundary $\partial \Omega$ and $f$ is an arbitrary holomorphic function in $\Omega$, continuous up to the boundary. Taking $n=1$ we obtain the classical Cauchy formula. In the same way one can establish other more complicated formulae associated with the names of mathematicians such as Fantappiè, Leray, Koppelman, Henkin, Ramirez, Øvrelid, Berndtsson, Andersson (cf. e.g. [1]).

3. A second order invariant Green's function. Let us put into play the partial differential operator

$$
\boldsymbol{\Delta}=\left(1-|z|^{2}\right)^{2} \Delta
$$

where $\Delta$ is, as before, the ordinary Laplacean. The operator $\Delta$ is primarily considered over the unit disc $\mathbb{D}$ and it is well known that it is invariant under conformal automorphisms of $\mathbb{D}$ (= Möbius maps of $\mathbb{D}$ onto itself). In fact, as noted already in the introduction, $\boldsymbol{\Delta}$ is nothing but the Laplace-Beltrami operator associated with the hyperbolic or Poincaré metric in $\mathbb{D}$-perhaps it might be called the Laplace-Beltrami-Poincaré operator.

Remark 1. More generally, one can consider the covariant operator

$$
\boldsymbol{\Delta}_{\alpha, \beta}=\left(1-|z|^{2}\right)^{2} \Delta+4 \alpha \bar{z} \frac{\partial f}{\partial z}+4 \beta z \frac{\partial f}{\partial \bar{z}}-4 \alpha \beta f .
$$

It is covariant in the sense that it intertwines with the group action

$$
f(z) \mapsto f(\omega(z))(e(z))^{\alpha}(\overline{e(z)})^{\beta} .
$$

Here $\alpha$ and $\beta$ are given numbers, usually real ones, and $\left(\begin{array}{l}a b \\ c d\end{array}\right)$ is an arbitrary unimodular pseudo-unitary matrix; pseudo-unitary means that $d=\bar{a}, c=\bar{b}$. It is clear that taking $\alpha=\beta=0$ gives back the previous special case, $\boldsymbol{\Delta}_{0,0}$ 
$=\boldsymbol{\Delta}$. Actually, two operators $\boldsymbol{\Delta}_{\alpha, \beta}$ and, say, $\boldsymbol{\Delta}_{\alpha_{1}, \beta_{1}}$ are similar provided $\alpha_{1}=\alpha+\kappa, \beta_{1}=\beta+\kappa$ for some number $\kappa$. Indeed, the corresponding intertwining map is of the form

$$
f(z) \mapsto f_{1}(z)=\left(1-|z|^{2}\right)^{-\kappa / 2}\left(1-|z|^{2}\right)^{-\kappa / 2} f(z) ;
$$

if $f(z)$ transforms with biweight $(\alpha+2, \beta+2)$ then $f_{1}(z)$ transforms with biweight $\left(\alpha_{1}+2, \beta_{1}+2\right)$. This follows from the well known relation

$$
1-|\omega(z)|^{2}=\frac{1-|z|^{2}}{|e(z)|^{2}} .
$$

We also refer to [15], Section 5, where the same transformation is considered in the context of general bounded symmetric domains. In Section 5 of the present paper we shall make use of the transformation (1) in a special case.

Returning to the operator $\boldsymbol{\Delta}$, we remark likewise that its iterates $\boldsymbol{\Delta}^{2}$, $\Delta^{3}$ etc. are conformally invariant. In this section we only consider the case of the square $\boldsymbol{\Delta}^{2}$ and, in particular, we wish to determine the corresponding invariant Green's function.

If $f$ is any radial function (smooth in $\mathbb{D} \backslash\{0\}$ ) then putting $|z|^{2}=r^{2}=t$ we may write

$$
\boldsymbol{\Delta} f=4(1-t)^{2}\left[t f^{\prime}\right]^{\prime}=4(1-t)^{2}\left[t f^{\prime \prime}+f^{\prime}\right] \quad \text { (radial part), }
$$

where the stroke ${ }^{\prime}$ is used to designate derivatives with respect to $t$. For later use we write down the formula

$$
\boldsymbol{\Delta}(f u)=\boldsymbol{\Delta} f \cdot u+8(1-t)^{2} t f^{\prime} u^{\prime}+f \boldsymbol{\Delta} u .
$$

We seek a basis for the solutions of the differential equation $\Delta^{2} f=0$, it can thus be viewed as a fourth order ordinary differential equation. In the case of $\boldsymbol{\Delta}$ itself such a basis is provided by the two functions $f_{0} \equiv 1$ and $g_{0}=\log t\left(=\log |z|^{2}\right)$. Taking them as part of our basis we need two more functions $f_{1}$ and $g_{1}$, say. We require $f_{1}$ to be regular at $t=0$ and to vanish there, and we seek $g_{1}$ of the form

$$
g_{1}=\log t \cdot f_{1}+\varphi_{1},
$$

where $\varphi_{1}$ is assumed to be regular at $t=0$ and to vanish there. Furthermore we require that

$$
\Delta f_{1}=4 f_{0}, \quad \Delta g_{1}=4 g_{0} .
$$

Then automatically $\boldsymbol{\Delta}^{2} f_{1}=0, \boldsymbol{\Delta}^{2} g_{1}=0$.

By the first half of (4) it is clear that we must have

$$
f_{1}=\int \frac{1}{t} \int \frac{1}{(1-t)^{2}}=\int \frac{1}{t} \frac{t}{1-t}=\int \frac{1}{1-t}=-\log (1-t) .
$$


Here we use the sign $\int$ to indicate the primitive, normalized to vanish at $t=0$. From (4) and (5) we obtain, using (3),

$$
\boldsymbol{\Delta} g_{1}=\boldsymbol{\Delta} f_{1} \cdot \log t+8(1-t)^{2} f_{1}^{\prime}+\boldsymbol{\Delta} \varphi_{1}
$$

or, if we take account of the second formula in (5) and that we know already $f_{1}$ (in particular that $f_{1}^{\prime}=1 /(1-t)$,

$$
\Delta \varphi_{1}=-8(1-t) \text {. }
$$

Integrating implies that

$$
\varphi_{1}=\int \frac{1}{t} \int \frac{-2(1-t)}{(1-t)^{2}}=-2 \int \frac{1}{t} \int \frac{1}{1-t}=2 \int \frac{\log (1-t)}{t}=-2 \operatorname{Li}_{2}(t),
$$

where $\mathrm{Li}_{2}$ stands for the dilogarithm (for the dilogarithm and related functions see the monograph [13]; cf. also [14] $\left.\left({ }^{1}\right)\right)$ :

$$
\operatorname{Li}_{2}(t):=-\int \frac{\log (1-t)}{t}=\sum_{n=1}^{\infty} \frac{t^{n}}{n^{2}}
$$

In particular, we note that $\operatorname{Li}_{2}(1)=\zeta(2)=\pi^{2} / 6$. Using (4) we finally obtain

$$
g_{1}=-\log t \cdot \log (1-t)-2 \mathrm{Li}_{2}(t) .
$$

We may summarize our result as follows.

Lemma 1. A basis for radial solutions (in $\mathbb{D} \backslash\{0\}$ ) of the differential equation $\boldsymbol{\Delta}^{2} f=0$ is given by the four functions

$f_{0} \equiv 1, \quad g_{0}=\log t, \quad f_{1}=-\log (1-t), \quad g_{1}=-\log t \cdot \log (1-t)-2 \operatorname{Li}_{2}(t)$, or, if we restore the variable $z$,

$$
\begin{gathered}
f_{0} \equiv 1, \quad g_{0}=\log |z|^{2}, \quad f_{1}=-\log \left(1-|z|^{2}\right), \\
g_{1}=-\log |z|^{2} \cdot \log \left(1-|z|^{2}\right)-2 \operatorname{Li}_{2}\left(|z|^{2}\right) .
\end{gathered}
$$

This basis is essentially unique, in the sense made precise in the above discussion.

Remark 2. We note that obviously

$$
g_{0}(t)+f_{1}(1-t)=0 .
$$

$\left({ }^{1}\right)$ We cannot resist pointing out here that, although the dilogarithm had been studied earlier by Euler and Landen, the very word was first used by the little known and nowadays (even in his home town Lund) not much esteemed Swedish mathematician Carl Johan Danielsson Hill (1793-1875) in his 1828 paper [10]. At some stage of our investigation, P. J. H. Hedenmalm suggested that we use the notation $\Delta$ in place of $\boldsymbol{\Delta}$. We were therefore somewhat amused when we later learnt that Hill in his 1830 dissertation [11] had introduced the Thor function for which he employed the notation $\mathcal{D}$. 
On the other hand, one has the following well known formula due to Euler (see [13], p. 5):

$$
\mathrm{Li}_{2}(t)=\frac{\pi^{2}}{6}-\log t \cdot \log (1-t)-\mathrm{Li}_{2}(1-t) .
$$

Therefore one sees that one also has

$$
g_{1}(t)+g_{1}(1-t)=-\frac{\pi^{2}}{3} .
$$

The beautiful identities (6) and (7) can be explained by a rather unexpected symmetry property of the equation $\boldsymbol{\Delta}^{2} f=0$, still for radial functions: it is invariant under the transformation $f(t) \mapsto f(1-t)$. This follows from the formula

$$
\Delta^{2}=s^{2}\left\{s^{2} t^{2} D^{4}+5 s t(s-t) D^{3}+2\left(s^{2}+t^{2}-4 s t\right) D^{2}-2(s-t) D\right\}
$$

which is readily verified by hand. In (8) we have put $s=1-t$ and $D=\frac{d}{d t}$. In particular, it follows thus that if we have two linearly independent solutions of $\boldsymbol{\Delta}^{2} f=0$, then we can construct out of them a full basis.

We are now looking for a radial fundamental solution $\mathbf{G}=\mathbf{G}(z)$, that is, we demand that $\boldsymbol{\Delta}^{2} \mathbf{G}=\delta$, where $\delta$ is the delta function at the origin. As $\mathbf{G}$ must be smooth at the origin, it must be a linear combination of the three functions $f_{0}, f_{1}$ and $g_{1}$, thus excluding $g_{0}=\log |z|^{2}$, which is not smooth there. Next, we require that $\mathbf{G}$ vanish for $|z|=1$. This excludes $f_{1}$ as well. Thus $G$ must be of the form

$$
\mathbf{G}=\mathbf{G}(t)=c \cdot\left[\log t \cdot \log \left(\frac{1}{1-t}\right)-2 \mathrm{Li}_{2}(t)+C\right],
$$

or, in terms of $z$,

$$
\mathbf{G}=\mathbf{G}(z)=c \cdot\left[\log |z|^{2} \cdot \log \left(\frac{1}{1-|z|^{2}}\right)-2 \operatorname{Li}_{2}\left(|z|^{2}\right)+C\right] .
$$

Looking at the expansion at the origin, $\mathbf{G}(t)=c \cdot(t \log t+\ldots)$, we convince ourselves that the outer constant must be $c=c_{2}=1 /(16 \pi)$ (see Section 2). To find the inner constant $C$ we make $|z|=1$. This gives $C=\pi^{2} / 3$. Thus we end up with the formula

$$
\mathbf{G}(z)=\mathbf{G}(z, 0)=\frac{1}{16 \pi} \cdot\left[\log |z|^{2} \cdot \log \left(\frac{1}{1-|z|^{2}}\right)-2 \operatorname{Li}_{2}\left(|z|^{2}\right)+\frac{\pi^{2}}{3}\right] .
$$

This is our sought Green's function at the origin. Using the invariance it is now easy to write down the corresponding formula for Green's function at any point $w \in \mathbb{D}$ : 
TheOREM 1. Green's function for the operator $\boldsymbol{\Delta}^{2}$ is given by

$$
\begin{aligned}
\mathbf{G}(z, w)= & \frac{1}{16 \pi} \cdot\left[\log \left|\frac{z-w}{1-z \bar{w}}\right|^{2} \log \frac{|1-z \bar{w}|^{2}}{\left(1-|z|^{2}\right)\left(1-|w|^{2}\right)}\right. \\
& \left.-2 \operatorname{Li}_{2}\left(\left|\frac{z-w}{1-z \bar{w}}\right|^{2}\right)+\frac{\pi^{2}}{3}\right] .
\end{aligned}
$$

Here we have used formula (10) of Section 1.

R e mark 3. This reminds of the formula for Green's function for the operator $\Delta$. Let us write for a moment the latter, omitting the constant $4 \pi$, as

$$
G(z, w)=\log \left|\frac{z-w}{1-z \bar{w}}\right|^{2}
$$

also put (essentially the logarithm of the Berezin kernel)

$$
H(z, w)=\log \frac{|1-z \bar{w}|^{2}}{\left(1-|z|^{2}\right)\left(1-|w|^{2}\right)} .
$$

Then we can write (9) in the conspicuous form

$$
\mathbf{G}(z, w)=\frac{1}{16 \pi} \cdot\left[G(z, w) \cdot H(z, w)-2 \operatorname{Li}_{2}(\exp G(z, w))+\frac{\pi^{2}}{3}\right] .
$$

From this we see, in particular, that (9) is not only Möbius invariant, but also conformally invariant. In other words, in view of the Riemann mapping theorem, we can state our result for any simply connected planar domain. There also arises the natural question of what can be said in the case of multiply connected domains. Let us mention that Green's function for the operator $\Delta^{2}$ (ordinary bi-Laplacean, not the invariant one!) in a circular annulus has been considered in [5].

R e mark 4 (positivity). Let us show that the function $\mathbf{G}(z, w)$ is positive in $\mathbb{D} \backslash\{w\}$; this is a small contribution to the Boggio-Hadamard problem (see Remark 2 in Section 1; cf. also [5]). It clearly suffices to take $w=0$. In other words, it suffices to show that the function $g_{1}=g_{1}(t)$ (recall that $\mathbf{G}=c\left(g_{1}+C\right)$ ) is monotone. Differentiating the relation $g_{1}=-\log t \cdot \log (1-t)-2 \operatorname{Li}_{2}(t)$ yields

$$
g_{1}^{\prime}=-\frac{\log (1-t)}{t}+\frac{\log t}{1-t}+2 \frac{\log (1-t)}{t}=\frac{\log (1-t)}{t}+\frac{\log t}{1-t}<0 .
$$

Thus $g_{1}$ is a decreasing function.

Remark 5. A Green's function usually solves a boundary problem, so what does our function $\mathbf{G}$ solve? Let $f$ be a smooth function with compact support in $\mathbb{D}$ and set 


$$
u(z)=\iint_{\mathbb{D}} \mathbf{G}(z, w) f(w) \frac{d x d y}{\left(1-|w|^{2}\right)^{2}} .
$$

Then the function $u$ is the unique solution of the equation $\Delta^{2} u=f$ subject to the boundary condition $u(z)=O(d(z) \log (1 / d(z))$, where $d(z)=$ $\operatorname{dist}(z, \partial \mathbb{D})$ is the distance to the boundary. Indeed, the most general solution of the equation $\boldsymbol{\Delta}^{2} u=0$ on $\mathbb{D}$ can be written as

$$
u(z)=\sum_{n=-\infty}^{\infty} u_{n}(r) r^{|n|} e^{n i \theta} \quad\left(z=r e^{i \theta}\right)
$$

where

$$
u_{n}(r)=a_{n}+b_{n} \sum_{k=1}^{\infty} \frac{r^{2 k}}{|n|+k} .
$$

When $r \rightarrow 1$, the last sum has the asymptotics $\sim \log (1 / d(z))$. Thus the condition $u(z)=O\left(d(z) \log (1 / d(z))\right.$ forces that $u_{n} \equiv 0$, and the claim concerning uniqueness follows.

4. On the higher order case. In this section we make an attempt to extend the result to the case of the higher powers $\boldsymbol{\Delta}^{m}, m>2$. However, we will obtain a conclusive result only for $m=3$.

Consider first, quite generally, the differential equation $\Delta^{m} f=0$, where $f$ is again a radial function. We seek a basis of solutions of the form $f_{0}, f_{1}, \ldots$ $\ldots, f_{m-1}, g_{0}, g_{1}, \ldots, g_{m-1}$ recursively determined by

(1) $\quad f_{0} \equiv 1, \quad g_{0}=\log t, \quad \Delta f_{i}=4 f_{i-1}, \quad \Delta g_{i}=4 g_{i-1} \quad(i>0)$.

We write

$$
g_{i}=f_{i} \log t+\varphi_{i}
$$

and require that $f_{i}$ and $\varphi_{i}$ be regular at $t=0$ and vanish there up to order $i-1$ :

$$
f_{i}=O\left(t^{i}\right), \quad \varphi_{i}=O\left(t^{i}\right) \quad(i>0) .
$$

As in Section 3, it now follows from (1) (and (3) in Section 3) that

$$
\Delta \varphi_{i}=4 \varphi_{i-1}-8(1-t)^{2} f_{i}^{\prime} .
$$

We must solve this equation, along with the third equation in (1), viz.

$$
\Delta f_{i}=4 f_{i-1} .
$$

Note that in (2)-(3) all functions are regular at $t=0$.

Consider for a while the general inhomogeneous equation $\Delta f=4 h$, where $f$ and $h$ are regular radial functions with Taylor developments

$$
f=a_{0}+a_{1} t+a_{2} t^{2}+\ldots \text { and } h=c_{0}+c_{1} t+c_{2} t^{2}+\ldots
$$

We must determine $f$ when $h$ is given. Inserting into the equation we find the recursion 


$$
\begin{aligned}
a_{1} & =c_{0}, \\
-2 a_{1}+4 a_{2} & =c_{1}, \\
(n-1)^{2} a_{n-1}-2 n^{2} a_{n}+(n+1)^{2} a_{n+1} & =c_{n} \quad(n>1) ;
\end{aligned}
$$

thus $a_{0}$ remains undetermined and we may always take it to be zero. (Putting $a_{-1}=0$ we can achieve that the equation on the last line is formally true for all $n=0,1,2, \ldots)$ This gives

$$
a_{1}=c_{0}, \quad a_{2}=\frac{1}{4}\left(2 c_{0}+c_{1}\right), \quad a_{3}=\frac{1}{9}\left(3 c_{0}+2 c_{1}+c_{2}\right) \quad \text { etc. }
$$

and, quite generally,

$$
a_{n}=\frac{1}{n^{2}} \sum_{k=0}^{n-1}(n-k) c_{k} \quad(n>0) .
$$

We also note that if $c_{0}=c_{1}=\ldots=c_{i-2}=0$ then $a_{0}=a_{1}=\ldots=a_{i-1}=0$, that is, if $h=O\left(t^{i-1}\right)$ then $f=O\left(t^{i}\right)$. In particular, it is clear that iterating the transformation (4) we obtain, starting with $f_{1} \equiv 1$, the coefficients of $f_{1}, f_{2}, \ldots$ solving the recursion (3).

EXAMPLE 1. Taking $c_{0}=1, c_{1}=c_{2}=\ldots=0$ gives $a_{1}=1, a_{2}=\frac{1}{2}$, $a_{3}=\frac{1}{3}$ etc.; in other words, we recover the Taylor expansion of the function $f_{1}=-\log (1-t)$. Continuing, if we take instead $c_{0}=0, c_{1}=1, c_{2}=\frac{1}{2}$, $c_{3}=\frac{1}{3}$ etc. we get

$$
a_{1}=0, \quad a_{n}=\frac{1}{n^{2}} \sum_{k=1}^{n-1}(n-k) \frac{1}{k} \quad(n>1) .
$$

The last equality may also be written as

$$
a_{n}=\frac{1}{n} \sum_{k=1}^{n-1} \frac{1}{k}-\frac{n-1}{n^{2}}=\frac{1}{n} \sum_{k=1}^{n-1} \frac{1}{k}-\frac{1}{n}+\frac{1}{n^{2}} .
$$

Thus we find that

$$
f_{2}=\frac{1}{2} \log ^{2}(1-t)+\log (1-t)+\operatorname{Li}_{2}(t) .
$$

Here we have used the fact that

$$
\log (1-t)=-\sum_{n=1}^{\infty} \frac{t^{n}}{n}, \quad \log ^{2}(1-t)=2 \sum_{n=2}^{\infty} \sum_{k=1}^{n-1} \frac{1}{k} \frac{t^{n}}{n} .
$$

Let us now turn our attention to formula (2). Consider quite generally the equation $\boldsymbol{\Delta} f=4 h-8(1-t)^{2} j^{\prime}$, where the function $j$ has the Taylor coefficients $d_{0}, d_{1}, \ldots$ :

$$
j=d_{0}+d_{1} t+d_{2} t^{2}+\ldots,
$$

letting $a_{0}, a_{1}, \ldots$ and $c_{0}, c_{1}, \ldots$ be those of $f$ and $h$ respectively. As before, we obtain, as a generalization of (4), 


$$
a_{n}=\frac{1}{n^{2}} \sum_{k=0}^{n-1}(n-k) c_{k}-2 \frac{d_{n}}{n} \quad(n>0) .
$$

EXAMPLE 2. Taking $c_{0}=c_{1}=\ldots=0, d_{0}=0, d_{1}=1, d_{2}=\frac{1}{2}$, $d_{3}=\frac{1}{3}$ etc., corresponding to $\varphi_{0}=0$ and $f_{1}=-\log (1-t)$ respectively, gives $a_{n}=-2 / n^{2}(n>0)$, which corresponds to $\varphi_{1}=-2 \operatorname{Li}_{2}(t)$, as we knew already. Next we take

$$
c_{0}=0, \quad c_{k}=-\frac{2}{k^{2}} \quad(k=1,2, \ldots), \quad d_{k}=\frac{1}{k} \sum_{j=1}^{k-1} \frac{1}{j}-\frac{1}{k}+\frac{1}{k^{2}} .
$$

This, apparently, corresponds to admitting the functions $h=\varphi_{1}=-2 \mathrm{Li}_{2}(t)$ and $j=f_{2}=\frac{1}{2} \log ^{2}(1-t)+\log (1-t)+\operatorname{Li}_{2}(t)$ (cf. Example 1 ultra). After some manipulations, one finds

$$
\varphi_{2}=2\left[\operatorname{Li}_{2}(t) \cdot \log (1-t)+\mathrm{M}_{3}(t)+\mathrm{Li}_{2}(t)-\mathrm{Li}_{3}(t)\right] .
$$

Here $\mathrm{Li}_{3}$ stands for the trilogarithm, defined by the formula (cf. [13], Chap. 6)

$$
\mathrm{Li}_{3}(t):=\int \frac{\mathrm{Li}_{2}(t)}{t}=\sum_{n=1}^{\infty} \frac{t^{n}}{n^{3}},
$$

while the function $\mathrm{M}_{3}$ is defined as

$$
\mathrm{M}_{3}(t)=\int \frac{\log ^{2}(1-t)}{t} .
$$

(It is easy to verify a posteriori by differentiation that $\varphi_{2}$ is indeed the function that we were looking for, so we will not go into details.)

Remark 1 . The function $M_{3}$ is closely related to a function introduced by Kummer and denoted by $\Lambda_{3}$ in [13] (see e.g. formula (6.114)). It is also in a simple way connected with the trilogarithm. To see this put $s=1-t$. Then one has

$$
\mathrm{M}_{3}(t)=\int_{0}^{t} \frac{\log ^{2}(1-t)}{t} d t=K-\int_{0}^{s} \frac{\log ^{2} s}{1-s} d s
$$

where we have put

$$
K=\int_{0}^{1} \frac{\log ^{2}(1-t)}{t} d t\left(^{2}\right) .
$$

Integrating the last integral twice by parts gives

$\left({ }^{2}\right)$ Using Mathematica we found the numerical value $K=2.4041138063191885708$. In fact, setting $t=0$ in the formula below for the function $\mathrm{M}_{3}$ one sees that indeed $K=2 \zeta(3)$ (=2. Apéry's constant); this was also kindly pointed out to us by Prof. Lewin (personal communication). 


$$
\begin{aligned}
\int_{0}^{s} \frac{\log ^{2} s}{1-s} d s & =-\log (1-s) \cdot \log ^{2} s+2 \int_{0}^{s} \frac{\log (1-s)}{s} \cdot \log s d s \\
& =-\log (1-s) \cdot \log ^{2} s-2 \operatorname{Li}_{2}(s) \cdot \log s+2 \int_{0}^{s} \frac{\operatorname{Li}_{2}(s)}{s} d s .
\end{aligned}
$$

Thus one finds

$$
\mathrm{M}_{3}(t)=K+\log t \cdot \log ^{2}(1-t)+2 \mathrm{Li}_{2}(1-t) \cdot \log (1-t)-2 \mathrm{Li}_{3}(1-t)
$$

this formula seems to be essentially due to Kummer (cf. [13], formula $(6.113))$.

The information contained in Examples 1 and 2 may be summarized as follows.

SCholium 1. The differential equation $\boldsymbol{\Delta}^{3} f=0$ has a basis of radial solutions $\left\{f_{0}, f_{1}, f_{2}, g_{0}, g_{1}, g_{2}\right\}$, where $g_{i}=f_{i} \log t+\varphi_{i}(i=0,1,2)$ and

$$
\begin{gathered}
f_{0} \equiv 1, \quad f_{1}=-\log (1-t), \\
f_{2}=\frac{1}{2} \log ^{2}(1-t)+\log (1-t)+\operatorname{Li}_{2}(t), \\
\varphi_{0}=0, \quad \varphi_{1}=-2 \operatorname{Li}_{2}(t), \\
\varphi_{2}=2\left[\operatorname{Li}_{2}(t) \cdot \log (1-t)+\mathrm{M}_{3}(t)+\operatorname{Li}_{2}(t)-\operatorname{Li}_{3}(t)\right],
\end{gathered}
$$

where again, generally speaking, $\operatorname{Li}_{m}(m=2,3, \ldots)$ stands for the polylogarithm, while the function $\mathrm{M}_{3}$ is defined by formula (6).

From Scholium 1 we may read off the boundary behavior of these functions; in particular,

$f_{0}(0)=1 \quad$ (trivial!), $\quad f_{1}(t)=t+O\left(t^{2}\right), \quad f_{2}(t)=\frac{1}{4} t^{2}+O\left(t^{3}\right) \quad$ as $t \rightarrow 0$.

In fact, it is easily seen that this is a special case of a general result:

$$
f_{m}(t)=\frac{1}{(m !)^{2}} t^{m}+O\left(t^{m+1}\right) \quad \text { as } t \rightarrow 0 .
$$

The above computations can, in principle, be continued to yield a basis of radial solutions also in the case of the equation $\Delta^{m} f=0$ when $m>3$. However, it seems that in general it is not very easy to express the functions $f_{i}$ and $\varphi_{i}$ in terms of known special functions. (Maybe one should simply promote these functions to the level of special functions of their own right!)

Returning to our original question concerning the determination of the Green's function $\mathbf{G}=\mathbf{G}_{m}$ of the operator $\boldsymbol{\Delta}^{m}(m>2)$, it is, in view of (8) and the other properties of our basis, natural to seek its radial part in the form

$$
\mathbf{G}_{m}(t)=(m !)^{2} c_{m}\left(g_{m-1}+A_{0} f_{0}+A_{1} f_{1}+\ldots+A_{m-1} f_{m-1}\right),
$$


where $c_{m}$ is the constant determined in Section 2 and $A_{0}, A_{1}, \ldots A_{m-1}$ are certain yet undetermined coefficients. At least it is clear that we have the crucial relation $\boldsymbol{\Delta}^{m} \mathbf{G}_{m}=\delta$.

EXAMPLE 3. If $m=1$, then we have $A_{0}=0$, which agrees with $\mathbf{G}_{1}=$ $(4 \pi)^{-1} \log t\left(=G_{1}\right)$. If $m=2$, we take $A_{0}=C=\pi^{2} / 3$ and $A_{1}=0$, which likewise agrees with the result in Section 3.

In general, the $A_{i}$ 's have to be chosen in such a way that the function vanishes at $t=1$ up to a suitable (maximum) order.

Let us look at the case $m=3$. Then (9) takes the form

$$
\mathbf{G}_{3}(t)=(3 !)^{2} c_{3}\left(g_{2}+A_{0} f_{0}+A_{1} f_{1}+A_{2} f_{2}\right) .
$$

Put once more $s=1-t$, so that $s=0$ if $t=1$. From (7) we infer that

$$
\begin{array}{cl}
f_{0} \equiv 1 \quad \text { (again trivial), } \quad f_{1}=-\log s, & f_{2}=\frac{1}{2} \log ^{2} s+O(\log s) ; \\
\varphi_{2}=\frac{\pi^{2}}{3} \log s+K^{\prime}+o(1) & \text { as } s \rightarrow 0 .
\end{array}
$$

where $K^{\prime}=2\left\{K+\pi^{2} / 6-\zeta(3)\right\}$ with $K$ as in Remark $1\left(^{3}\right)$. We now choose

$$
A_{0}=-K^{\prime}, \quad A_{1}=\frac{\pi^{2}}{3}, \quad A_{2}=0 .
$$

Then we achieve $G_{3}=O\left(s \log ^{2} s\right)$ as $s \rightarrow 0$ or $t \rightarrow 1$. To sum up, we have thus established the following result.

THEOREM 1. The partial differential operator $\boldsymbol{\Delta}^{3}$ has invariant Green's function $\mathbf{G}_{3}=\mathbf{G}_{3}(z, w)$, whose radial part is given by

$$
\mathbf{G}_{3}(t)=\frac{1}{64 \pi}\left(f_{2} \cdot \log t+\varphi_{2}-K^{\prime} f_{0}+\frac{\pi^{3}}{3} f_{1}\right),
$$

where the functions $f_{0}, f_{1}, f_{2}, \varphi_{2}$ are provided by formula (7), while the constant $K^{\prime}$ is as in (10). One has, for $w$ fixed,

$$
\mathbf{G}_{3}(z, w)=O\left(d(z) \log ^{2} d(z)\right),
$$

where $d(z)$ is the distance to the boundary.

We expect that a similar result holds also for $m>3$, that is, we have an invariant Green's function $\mathbf{G}_{m}$ satisfying $\mathbf{G}_{m}(z)=O\left(d(z) \log ^{m-1} d(z)\right)$ at the boundary. But as we so far do not possess a sufficiently explicit representation of the functions in the corresponding basis, we are unable to prove it. At any rate, in view of the fact that the present paper is already quite long, it seems not to be advisable to try and include anything more about $m>3$ here.

5. Connection between $\Delta^{m}$ and the invariant Laplacean $\Delta$. In this section we shall relate the iterates $\Delta^{m}(m=0,1, \ldots)$ of the ordinary

$\left({ }^{3}\right)$ In view of footnote $\left({ }^{2}\right)$ one has $K^{\prime}=2(\zeta(3)+\zeta(2))$. 
Laplacean $\Delta$ to the invariant one $\boldsymbol{\Delta}$. This is done by invoking the operator

$$
L_{m}=\left(1-|z|^{2}\right)^{m+1} \Delta^{m}\left(1-|z|^{2}\right)^{m-1} .
$$

It is readily seen (using what was said in Remark 1 in Section 3) that this is an invariant operator.

Now we recall a general fact about symmetric spaces, viz. that the algebra of invariant differential operators on a symmetric space of rank $r$ is isomorphic to a polynomial algebra in $r$ variables (cf. [9], Section II.4.3, especially Proposition II.4.11). The disc $\mathbb{D}$ is a (Hermitean) symmetric space of rank one, and in this case one can take the single generator to be the invariant Laplace-Beltrami operator $\boldsymbol{\Delta}$. Therefore we know a priori that $L_{m}$ must be a polynomial in $\boldsymbol{\Delta}$. We wish to identify this polynomial. Here is the result:

THEOREM 1. We have

$$
L_{m}=p_{m}(\boldsymbol{\Delta})
$$

where $p_{m}$ is the polynomial

$$
p_{m}(\lambda)=\prod_{j=1}^{m}(\lambda-4 j(j-1)) .
$$

Thus, in particular,

$$
p_{1}(\lambda)=\lambda, \quad p_{2}(\lambda)=\lambda^{2}-8 \lambda, \quad p_{3}(\lambda)=\lambda^{3}-32 \lambda^{2}+192 \lambda \quad \text { etc. }
$$

R e mark 1. In [15] the case of the corresponding compact symmetric space, i.e. the Riemann sphere $S^{2}$, was considered. It was proved that the operator $D^{m} \bar{D}^{m}$ can be expressed as a polynomial of the "compact" analogue of the operator $\boldsymbol{\Delta}_{(\alpha, 0)}$ (cf. Remark 1 in Section 3) on the sphere. Here $\bar{D}$ is the invariant Cauchy-Riemann operator on $S^{2}$,

$$
\bar{D}=\left(1+|z|^{2}\right)^{2} \frac{\partial}{\partial \bar{z}},
$$

and $D$ is its adjoint (it depends on which metric we are using, that is, the value of $\alpha$; see Section 6). But a corresponding result is true also in the present non-compact situation and it then turns out that if $\alpha=0$ one obtains exactly the same polynomial as in Theorem 1 . One only has to make formal changes, replacing the expression $1+|z|^{2}$, related to the Bergman kernel, by $1-|z|^{2}$, etc. Thus we can draw the conclusion that we have a relation of the type $L_{m}=D^{m} \bar{D}^{m}$. In this case the definition reads:

$$
\bar{D}=\left(1-|z|^{2}\right)^{2} \frac{\partial}{\partial \bar{z}}
$$


The proof of Theorem 1 given below is not very hard but before turning to it let us have a look at two special cases: $m=1$, which is trivial, and $m=2$, because treating this case directly reveals one interesting feature.

ExAmple $1(m=1)$. It is clear that $L_{1}=\boldsymbol{\Delta}=\left(1-|z|^{2}\right)^{2} \Delta$, which agrees with $p_{1}(\lambda)=\lambda$.

EXAMPle $2(m=2)$. It is convenient to put $w=1-|z|^{2}$. Then we can write

$$
L_{2}=w^{3} \Delta^{2} w=w^{2} \cdot w \Delta^{2} w .
$$

We shall also use the same letter $w$ to denote the operation of multiplication by the function $w$. Let us further invoke the Euler operator $\mathfrak{E}$, viz.

$$
\mathfrak{E}=z \frac{\partial}{\partial z}+\bar{z} \frac{\partial}{\partial \bar{z}} .
$$

Then one has the relations

$$
\begin{aligned}
& \Delta w=w \Delta-4 \mathfrak{E}-4, \quad[w, \Delta]=4(\mathfrak{E}+1), \\
& \Delta \mathfrak{E}=\mathfrak{E} \Delta+2 \Delta, \quad \text { or } \quad[\mathfrak{E}, \Delta]=-2 \Delta, \\
& \mathfrak{E} w=w \mathfrak{E}+2 w-2, \quad[\mathfrak{E}, w]=2(w-1) ;
\end{aligned}
$$

the bracket [] is used to designate the commutator of operators. Using the formulae (3) we find

$$
\begin{aligned}
w \Delta^{2} w & =(\Delta w+4 \mathfrak{E}+4)(w \Delta-4(\mathfrak{E}+1)) \\
& =\Delta w^{2} \Delta-4 \Delta w(\mathfrak{E}+1)+4(\mathfrak{E}+1) w \Delta-16(\mathfrak{E}+1)^{2} \\
& =w^{-2} \Delta^{2}-4(\Delta w \mathfrak{E}-\mathfrak{E} w \Delta)-4(\Delta w-w \Delta)-16\left(\mathfrak{E}^{2}+2 \mathfrak{E}+1\right) .
\end{aligned}
$$

The penultimate term in this formula is equal, again in view of (3), to $-4(-4 \mathfrak{E}-4)=16(\mathfrak{E}+4)$. In order to treat the third term from the end we write

$$
\begin{aligned}
\mathfrak{E} w \Delta & =\mathfrak{E} \Delta w+4 \mathfrak{E}^{2}+4 \mathfrak{E}=\Delta \mathfrak{E} w-2 \Delta w+4 \mathfrak{E}^{2}+4 \mathfrak{E} \\
& =\Delta w \mathfrak{E}+2 \Delta w-2 \Delta-2 \Delta w+4 \mathfrak{E}^{2}+4 \mathfrak{E} .
\end{aligned}
$$

Inserting this information into (4) gives finally $L_{2}=L_{1}^{2}-8 L_{1}$, in agreement with $p_{2}(\lambda)=\lambda^{2}-8 \lambda$.

Remark 2. The left equations in (3) express the fact that the operators $\Delta, w$ and $\mathfrak{E}$ span together with the identity operator 1 a fourdimensional Lie algebra. (Indeed, if we are willing to replace $\mathfrak{E}$ by $\mathfrak{E}_{1}=\mathfrak{E}+1$ and $w$ by $w_{1}=w-1$, these equations become even somewhat simpler and we have the constituting relations of $\mathfrak{s l}(2)$.) So, in principle, our theorem can be viewed as a result about an enveloping algebra. However, we were not successful in our attempt to prove Theorem 1 along these lines. 
Proof of Theorem 1. It is convenient to introduce the variable $s=1-t=1-|z|^{2}$. Then the radial part of $\boldsymbol{\Delta}$ is given by

$$
\boldsymbol{\Delta} f=4 s^{2}\left((1-s) f^{\prime}\right)^{\prime} \quad \text { if } f=f(s) .
$$

We observe that $\boldsymbol{\Delta} s^{k}=4 k(k-1) s^{k}-4 k^{2} s^{k+1}$. Iterating from $k=1$ to $k=m$ gives

$$
\prod_{k=1}^{m}[\boldsymbol{\Delta}-4 k(k-1)] s=(-1)^{m} m !^{2} 4^{m} s^{m+1} .
$$

Next, if $P(s)$ is any polynomial in $s$ of degree $p$ and leading coefficient $c$, then $\Delta P(s)$ is a polynomial in $s$ of degree $p-1$ and with leading coefficient $-4 p^{2} c$. Setting $P(s)=s^{m}$ and iterating $m$ times, we see that

$$
\Delta^{m} s^{m}=(-1)^{m} m !^{2} 4^{m} s^{0},
$$

that is,

$$
\left(s^{m+1} \Delta^{m} s^{m-1}\right) s=(-1)^{m} m !^{2} 4^{m} s^{m+1},
$$

which is the same right hand side as in the formula (5).

Finally, using the covariance we infer that the relation

$$
s^{m+1} \Delta^{m} s^{m-1} f=\prod_{k=1}^{N}[\boldsymbol{\Delta}-4 k(k-1)] f
$$

holds not only for $f=s$, but for any function $f$.

Alternative proof (via the upper halfplane). By conformal invariance it suffices to prove the corresponding result for the upper (Poincaré) halfplane $\{z: y>0\}$. The rôle of the invariant Laplacean in the disc is then taken over by the differential operator $4 y^{2} \Delta$, for which we retain the same notation $\boldsymbol{\Delta}$, while we put

$$
L_{m}=(2 y)^{m+1} \Delta^{m}(2 y)^{m-1} .
$$

Thus we have to establish the validity of (1) with this new interpretation of $L_{m}$ and $\boldsymbol{\Delta}$. Let us work with the functions $y^{\sigma}$, where $\sigma$ stands for any complex number. These functions are eigenfunctions of $\boldsymbol{\Delta}$ with eigenvalue $\lambda$ :

$$
\boldsymbol{\Delta} y^{\sigma}=\lambda y^{\sigma} \quad \text { with } \quad \lambda=4 \sigma(\sigma-1) .
$$

Using this it is easy to see that

$$
L_{m} y^{\sigma}=p_{m}(\lambda) y^{\sigma}
$$

and the proof is completed by an argument similar to the one used in the previous proof. (Using further a simple density argument, this proof works even without the a priori knowledge that $L_{m}$ must be a polynomial in $\boldsymbol{\Delta}$, which, as we know, is a deep result on invariant partial differential operators on symmetric spaces.) 
R e mark 3 (on Green's functions). Theorem 1 above suggests the question of what can be said about differential operators of the form $p(\boldsymbol{\Delta})$, where $p$ is an arbitrary polynomial, in general, and Green's functions of such operators in particular. For instance, in view of the result in Remark 4 in Section 3 it is a legitimate question to ask for which polynomials $p$ it is true that the corresponding Green's function is positive. To fix ideas let us first assume that $p$ is a polynomial of degree 2 with two distinct roots $a$ and $b$, i.e. $p(\lambda)=(\lambda-a)(\lambda-b)$-thus the non-degenerate situation. Then, according to a remark by Alexander Weinstein (cf. [17], p. 136), every solution $u$ to the partial differential operator

$$
(\boldsymbol{\Delta}-a)(\boldsymbol{\Delta}-b) u=0
$$

can be written as a sum $u=u_{a}+u_{b}$, where $(\boldsymbol{\Delta}-a) u_{a}=0$ and $(\boldsymbol{\Delta}-b) u_{b}=0$. Therefore, in principle, the problem is reduced to degree one. Let us look at radial solutions of any of these two equations, that is, functions $f=f(t)$, with $t=r^{2}=|z|^{2}$ as before, such that

$$
(1-t)^{2}\left[t f^{\prime \prime}+f^{\prime}\right]-\lambda f=0,
$$

where $4 \lambda$ stands for any of the numbers $a$ or $b$. If we put $s=1-t$, we can write this equation as

$$
s^{2}(s-1) f^{\prime \prime}+s^{2} f^{\prime}+\lambda f=0,
$$

which is again a special case of the more general ordinary differential equation

$$
s^{2}(s-1) f^{\prime \prime}+[(a+b+1) s+(\alpha+\beta-1)] s f^{\prime}+(a b s-\alpha \beta) f=0,
$$

namely the special case $a=b=0, \alpha, \beta=1 / 2 \pm \varrho$, where we have put $\lambda+1 / 4=\varrho^{2}$. It follows from the marvellous book [12], (19), p. 425, that the general solution of equation (6) is of the form

$$
f=s^{\alpha} y(a+\alpha, b+\alpha ; \alpha-\beta+1 ; s),
$$

where $y(, ; ;)$ is an ad hoc notation for the (generic) hypergeometric function. It follows from (7) that in our case one has

$$
f(s)=s^{1 / 2+\varrho} y(1 / 2+\varrho, 1 / 2+\varrho ; 1+2 \varrho ; s)
$$

or, equivalently, in terms of the variable $t$,

$$
f(t)=(1-t)^{1 / 2+\varrho} y(1 / 2+\varrho, 1 / 2+\varrho ; 1 ; t) .
$$

In particular, we thus have the regular (at $s=0$ or $t=1$ ) solution $f_{1}$ given by

$$
f_{1}(s)=s^{1 / 2+\varrho} F(1 / 2+\varrho, 1 / 2+\varrho ; 1+2 \varrho ; s)
$$

and also the regular (at $t=0$ ) solution $f_{2}$ given by

$$
f_{2}(t)=(1-t)^{1 / 2+\varrho} F(1 / 2+\varrho, 1 / 2+\varrho ; 1 ; t),
$$


where $F={ }_{2} F_{1}(, ; ;)$ is the standard notation for the Gauss hypergeometric function. Note that if $\lambda=0$, in which case we can take $\varrho=1 / 2$, the former formula gives the function $\log t$ or $\mathbf{1}$. Thus, summarizing our discussion and abstracting, we may conclude that Green's function also in the general case ( $p$ an arbitrary polynomial) may be expressed in terms of known "higher transcendental functions", hypergeometric functions or degenerate cases of them. As essentially an illustration of this fact we may mention that the dilogaritm $\operatorname{Li}_{2}(x)$ can be obtained from the function $F(\varepsilon, \varepsilon ; 1 ; x)$ by letting $\varepsilon \rightarrow 0$ (see [13], p. 30).

6. On the case of several complex variables. In this section we discuss to what extent the results of this paper can be generalized to the case of several complex variables. However, most of what we say here belongs to the category of general nonsense, but we hope to be able to return to this topic later on and do some more substantial work.

First of all we observe that the natural generalization of Bojarski's theorem [4] to higher dimensions is in the real, and involves conformal mapping. As biholomorphic transformations in several variables are conformal only in rare cases, we may conclude that the Euclidean Laplacean is not very productive from the present point of view: The results of Sections 1-2 have their natural extensions in the context of real variables. On the other hand, the invariant Laplacean of Sections 3-4 has an obvious counterpart in several variables: it is the Laplace-Beltrami operator in the ball of $\mathbb{C}^{n}$ equipped with the Bergman metric. Now the ball is a rank one symmetric domain. More generally, we could allow bounded symmetric domains of higher rank, but then we have the additional difficulty that the Laplace-Beltrami operator does not generate the whole algebra of invariant differential operators on the symmetric domain in question (cf. what was said about this in the second paragraph of Section 5). What remains of Section 5 is, however, the connection between invariant partial differential operators and the iterates of the invariant, or-perhaps better-covariant, Cauchy-Riemann operator $\bar{D}$ (in the one-dimensional case embodied in the result from [15] mentioned in Remark 1 of the said section). Let us elaborate on this point somewhat.

Consider first quite generally the situation when there is given a Kähler manifold $\Omega$ of dimension $n$ and an Hermitean vector bundle $E$ of rank $N$ over $\Omega$. In terms of local holomorphic coordinates $z=\left(z^{1}, \ldots, z^{n}\right)$ the metric on $\Omega$ is given by

$$
d s^{2}=h_{i j}(z) d z^{i} d \bar{z}^{j} .
$$

Similarly, picking a system of local trivializing sections $e_{\alpha}=e_{\alpha}(z)(\alpha=$ $1, \ldots, N)$ the metric on $E$ is given by certain coefficients $a_{\alpha \beta}=a_{\alpha \beta}(z)$ 
defined by the formula

$$
a_{\alpha \beta}(z)=\left(e_{\alpha}(z), e_{\beta}(z)\right)_{z},
$$

where $(\cdot, \cdot)_{z}$ denotes the inner product in the fiber $E_{z}$ of $E$ over the point with local coordinate $z$.

$\mathrm{N}$ ot e. Here and in what follows we use liberally the usual conventions of tensor calculus, including the summation convention.

Let $T^{\mathrm{c}} \Omega=T^{\prime} \Omega \oplus T^{\prime \prime} \Omega$ be the usual splitting of the complex tangent bundle of $\Omega$ into its holomorphic and anti-holomorphic components. Its dual, the complex cotangent bundle $T^{{ }^{*}} \Omega$, admits a corresponding splitting, giving in turn rise to the splitting $d=d^{\prime}+d^{\prime \prime}$ of the operation of exterior differentiation into its holomorphic and anti-holomorphic parts (thus $d^{\prime \prime}$ is just another notation for the $\bar{\partial}$-operator). By the F. Riesz lemma we have a canonical anti-holomorphic equivalence

$$
T^{\prime \prime *} \Omega \stackrel{\varrho}{\approx} T^{\prime} \Omega
$$

induced by the metric on $\Omega$. Composing with $d^{\prime \prime}$ we get a holomorphic linear mapping

(1) $\bar{D}=\varrho \circ d^{\prime \prime}: E \rightarrow T^{\prime} \Omega \otimes E \quad$ ("covariant" Cauchy-Riemann operator).

Explicitly: In terms of the local data just introduced we have

$$
\bar{D} f=h^{i j} \frac{\partial f^{\alpha}}{\partial \bar{z}^{j}} \partial_{i} \otimes e_{\alpha}, \quad \text { with } \partial_{i}=\frac{\partial}{\partial z^{i}} .
$$

Here $h^{i j}$ denotes the inverse of the matrix $h_{i j}$, while $f$ stands for any smooth section of $E$ with local coefficients $f^{\alpha}=f^{\alpha}(z)(\alpha=1, \ldots, N)$. If we restrict $f$ to be square integrable with respect to the Kähler (or Liouville) measure $\mu$ on $\Omega$, we may view $\bar{D}$ as a linear operator on the Hilbert space $L^{2}(\Omega, \mu, E)$. Therefore we can put into play its adjoint $D$,

$$
D: T^{\prime} \Omega \otimes E \rightarrow E .
$$

Explicitly:

$$
D g=a^{\alpha \beta} \frac{1}{\operatorname{det} h} \partial_{j}\left(\operatorname{det} h a_{\gamma \beta} g^{j \gamma}\right) e_{\alpha} .
$$

Here det $h$ stands for the determinant of the matrix $h_{i j}$, while $g$ is any smooth section of the bundle $T^{\prime} \Omega \otimes E$ with local coefficients $g^{j \alpha}, a^{\alpha \beta}$ being the inverse of the matrix $a_{\alpha \beta}$.

One virtue of the above construction of the Cauchy-Riemann operator $\bar{D}$ is that it can be iterated. Starting with a section $f$ of $E$ and having formed $\bar{D} f$, which is a section of $T^{\prime} \Omega \otimes E$, we apply the same construction to the case when the bundle $E$ is replaced by $T^{\prime} \Omega \otimes E$. This produces a section, written $\bar{D}^{2} f$, of the latter section. In the same way we can define $\bar{D}^{3}, \bar{D}^{4}, \ldots$ 
We can likewise define iterates of the adjoint operator: $D^{2}, D^{3}, \ldots$ The local expressions for these iterated operators are given by formulae which are straightforward generalizations of our previous (2) and (3), only many more indices are involved. We can also form, for each $m=1,2, \ldots$, the operator $L_{m}=D^{m} \bar{D}^{m}$. It is clear that $L_{m}: E \rightarrow E$. In particular, if $E$ is the trivial line bundle, then $E=\mathbb{C}, N=1$, and $L_{m}$ thus turns out to be a scalar operator.

Let us now apply the above to the case when $\Omega$ is an Hermitean symmetric space, in particular a bounded symmetric domain, denoting by $\mathfrak{G}$ the corresponding group of biholomorphic automorphisms. We also assume that $\mathfrak{G}$ operates by metric preserving transformations on $E$. Then it is clear that the operators $\bar{D}$ and $D$ intertwine with the group actions and so do their iterates and other composites. (This means, in particular, that we are allowed to remove the quotes around the word "covariant" in formula (1).) Likewise, it transpires that the operator $L_{m}$ is covariant, invariant if $E=\mathbb{C}$. This is the generalization of the corresponding operator in Section 5 that we have in mind.

Problem 6.1. Restricting ourselves to the scalar case $(E=\mathbb{C})$ it is now natural to ask what is the bearing of the operators $L_{m}$ to the general invariant partial differential operator on our Hermitean symmetric space $\Omega$. Thus, in particular: Is it true that the operators $L_{m}(m=1,2, \ldots)$ generate the algebra of all invariant partial differential operators on $\Omega$ ? Ideally, one might even hope that, if $r$ denotes the rank of $\Omega$, the first $r$ of them might suffice.

Acknowledgments. The authors are indebted to Per Jan Håkan Hedenmalm for useful information connected with this research.

\section{References}

[1] B. Berndtsson and M. Anders son, Henkin-Ramirez formulas with weight factors, Ann. Inst. Fourier (Grenoble) 32 (3) (1982), 91-110.

[2] T. B o g g i o, Sull'equilibrio delle piastre elastiche incastrate, Atti Reale Accad. Lincei 10 (1901), 197-205.

[3] -, Sulle funzioni di Green d'ordine m, Rend. Circ. Mat. Palermo 20 (1905), 97-135.

[4] B. B ojarski [B. Boyarski1], Remarks on polyharmonic operators and conformal mappings in space, in: Trudy Vsesoyuznogo Simpoziuma v Tbilisi, 21-23 Aprelya 1982 g., 49-56 (in Russian).

[5] M. Engliš and J. P e etre, A Green's function for the annulus, Ann. Mat. Pura Appl., to appear.

[6] B. Gust af s s o n and J. P e etre, Notes on projective structures on complex manifolds, Nagoya Math. J. 116 (1989), 63-88.

[7] W. K. Hay man and B. Kore $\mathrm{nblum}$, Representation and uniqueness of polyharmonic functions, J. Anal. Math. 60 (1993), 113-133. 
[8] P. J. H. Hed e n m a lm, A computation of Green functions for the weighted biharmonic operators $\Delta|z|^{-2 \alpha} \Delta$, with $\alpha>-1$, Duke Math. J. 75 (1994), 51-78.

[9] S. Helg as o n, Groups and Geometrical Analysis, Academic Press, Orlando, 1984.

[10] C. J. Hill, Über die Integration logarithmisch-rationaler Differentiale, J. Reine Angew. Math. 3 (1828), 101-159.

[11] - Specimen exercitii analytici, functionem integralem $\int_{0}^{x} \frac{d x}{x} L\left(1+2 x C \alpha+x^{2}\right)$ $=\mathcal{D} x$ tum secundum amplitudinem, tum secundum modulem comparandi modum exhibentis, Typis Berlingianis, Londinii Gothorum (三 Lund), 1830.

[12] E. K a m ke, Handbook of Ordinary Differential Equations, Nauka, Moscow, 1971 (in Russian).

[13] L. Lewin, Polylogarithm and Associated Functions, North-Holland, New York, 1981.

[14] L. Lewin (ed.), Structural Properties of Polylogarithms, Math. Surveys Monographs 37, Amer. Math. Soc., Providence, R.I., 1991.

[15] J. P e etr e and G. Z h a n g, Harmonic analysis on the quantized Riemann sphere, Internat. J. Math. Math. Sci. 16 (1993), 225-243.

[16] - - - A weighted Plancherel formula III. The case of the hyperbolic matrix ball, Collect. Math. 43 (1992), 273-301.

[17] H. W e y l, Ramifications, old and new, of the eigenvalue problem, Bull. Amer. Math. Soc. 56 (1950), 115-139; also in: Gesammelte Abhandlungen IV, 432-456.

Mathematical Institute

Academy of Sciences

Žitná 25

11567 Praha 1, Czech Republic

E-mail: englis@earn.cvut.cz
Department of Mathematics Lund University Box 118

S-22100 Lund, Sweden E-mail: jaak@maths.lth.se 